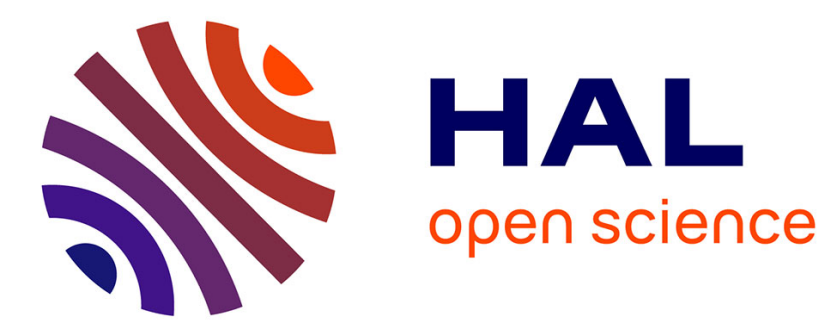

\title{
Thermal-wave slice tomography using wave field reconstruction
}

O. Pade, A. Mandelis

\section{To cite this version:}

O. Pade, A. Mandelis. Thermal-wave slice tomography using wave field reconstruction. Journal de Physique IV Proceedings, 1994, 04 (C7), pp.C7-99-C7-102. 10.1051/jp4:1994725 . jpa-00253253

\section{HAL Id: jpa-00253253 https://hal.science/jpa-00253253}

Submitted on 1 Jan 1994

HAL is a multi-disciplinary open access archive for the deposit and dissemination of scientific research documents, whether they are published or not. The documents may come from teaching and research institutions in France or abroad, or from public or private research centers.
L'archive ouverte pluridisciplinaire HAL, est destinée au dépôt et à la diffusion de documents scientifiques de niveau recherche, publiés ou non, émanant des établissements d'enseignement et de recherche français ou étrangers, des laboratoires publics ou privés. 


\title{
Thermal-wave slice tomography using wave field reconstruction
}

\author{
O. Pade(1) and A. Mandelis
}

Photothermal and Optoelectronic Diagnostics Laboratory, Department of Mechanical Engineering, University of Toronto, Toronto, Canada, MSS $1 A 4$

\begin{abstract}
A new matrix equation-based theoretical computation method for detection of subsurface defects by thermal-wave tomographic imaging is presented. The numerical calculations of the inverse problem were carried out using the Born approximation, however, the theoretical technique is capable of handling the general tomographic inverse case without recourse to the Born approximation.
\end{abstract}

\section{INTRODUCTION}

Thermal-wave Slice Diffraction Tomography (TSDT) was introduced as a photothermal imaging instrumentation technique [1-3] for the detection of sub-surface defects in solid material along crosssectional planes perpendicular to the laser-beam scanned surface. The first TSDT instrument was based on contacting photopyroelectric tomographic detection $[2,3]$ followed by ray-optic reconstruction of the cross-sectional thermal-wave image of the thermal diffusivity of the chosen slice [2]. The one-dimensional ray-optic based reconstruction technique was quite successful in illustrating the TSDT principle. However, using only ray-optic methods has many limitations, especially in highly dispersive wave fields, such as thermal waves. For this reason techniques familiar from $\mathrm{X}$-ray cross-sectional tomography, such as the recovery of a 2-D image from an oversampled 1-D projection, cannot be applied to TSDT with satisfactory image contrast, spatial resolution and low distortion. We therefore present a novel Computational Slice Diffraction Tomography based on the matrix methodology of Ref. [4]. The physical methodology is described and the computational technique allowing slice reconstruction and imaging is given for experimental situations involving both back-propagation as well as forward (transmission) thermal-wave data numerically manufactured from the solution to the direct problem (Helmholtz pseudo-wave equation $[5])$.

\section{MATHEMATICAL BACKGROUND}

In the case of a harmonic photothermal excitation of a region of space, the temperature oscillation is found to obey the Helmholtz pseudo-wave equation [4-6]:

$$
\left[\nabla^{2}+\tilde{k}^{2}(r)\right] T(r)=0 ; \tilde{k}(r)=(1-i)\left[\frac{\omega}{2 \alpha(r)}\right]^{1 / 2}
$$

(1) On leave from Rafael, P.O. Box 2250, Haifa 31021, Israel 
$\alpha(\mathrm{r})$ is the thermal diffusivity and $\omega$ is the angular frequency of the modulation of thelaser beam intensity. $\widetilde{k}(r)$ is the complex thermal wave number. Upon defining [5]

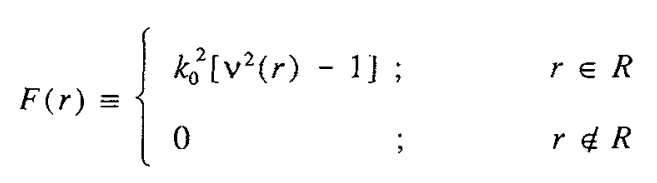

the diffusion (Helmholtz pseudo-wave) equation takes the form [6]

$$
\left(\nabla^{2}+\tilde{k}_{0}^{2}\right) T(r)=-F(r) T(r) .
$$

In equation (2) we defined $R$ to be the object region and

$$
v(r)=\left[\alpha_{0} / \alpha(r)^{1 / 2} ; \bar{k}_{0}=(1-i)\left(\frac{\omega}{2 \alpha_{0}}\right)^{1 / 2} \equiv k_{0} e^{-i \pi / 4}\right.
$$

$\alpha_{0}$ is the diffusivity of the homogeneous (reference) region surrounding the object region $R$. The full solution of Eq. (3) satisfies in three dimensions,

$$
T(r)=T_{i}(r)+\iiint_{S} G_{0}(r \mid \rho) F(\rho) T(\rho) d^{3} \rho-\iint_{B}\left[G_{0}(r \mid \rho) \frac{\partial T}{\partial n_{r}}(\rho)-T(\rho) \frac{\partial G_{0}}{\partial n_{r}}\right] d \rho
$$

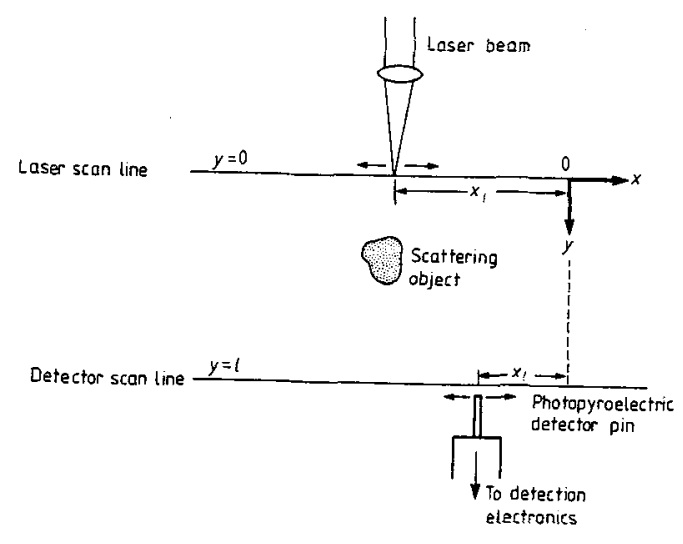

Fig. 1 Geometry for Thermal-Wave Slice Diffraction Tomography amenable to back-propagation (BP) and transmission ( $T$ ) detection. For each position of the laser beam waist on the line $y=0$, the photothermal detector measures the thermal-wave field characteristics (amplitude and phase) along the back surface $(y=l$ line; transmission) or along the front surface in the vicinity of the laser beam spot $\left(y=0\right.$ line; back-propagation). The region $0<y<y_{f}$ is the object region $S$. 
If the thermal-wave field $T\left(x, \mathrm{y}=y_{f}\right)$ is measured (transmission mode), and if

$$
T(r)=T_{i}(r)+T_{s}(r)
$$

then using Eq. (5) we obtain along the object region of the 2-D slice between $y=0$ and $y=y_{f}$ and between $x=x_{i}$ and $x=x_{f}$ :

$$
T_{s}\left(x, y_{f}\right)=\int_{x_{i}}^{x_{f} y_{0}} \int_{0} G_{0}\left[r\left(x, y_{f}\right) \mid \rho(\xi, \eta)\right] F[\rho(\xi, \eta)] T[\rho(\xi, \eta)] d \xi d \eta .
$$

If the thermal-wave field $T(x, y=0)$ is measured (back-propagated mode), then

$$
T_{s}(x, 0)=\int_{x_{i}}^{x_{j} y_{f}} \int_{0} G_{0}[r(x, 0) \mid \rho(\xi, \eta)] F[\rho(\xi, \eta)] T[\rho(\xi, \eta)] d \xi d \eta .
$$

Note that the surface integral contribution to the photothermal signal has been neglected without proof. The adequacy of this approximation on the accuracy of reconstruction of the object field function will be examined via the various artificial situations to be described in what follows. Thus, it will be shown $a$ posteriori that effective object field function reconstructions may be generated using only volume integral data in many situations corresponding to cases of practical interest and importance.

In Ref. [4] we show that Green's function for the two-dimensional thermal diffusion Helmholtz pseudowave Eq. (3) is

$$
G_{0}(|r-\rho|)=\frac{i}{4} H_{0}^{2}\left(e^{i \pi / 4} k_{0}|r-\rho|\right)
$$

where $H_{0}^{2}$ is the Hankel function of the second kind of order zero.

\section{THE COMPUTATIONAL METHOD FOR THE INVERSE PROBLEM}

The proposed technique is based on a special method of discretization of Eq. (5). Defining a matrix of coefficients $A$ by $A_{k m}=G_{0}\left(\left|r_{k}-\rho_{m}\right|\right)$, we have the following $n^{2} \times n^{2}$ system of linear equations:

$$
\text { Af }=t
$$

where $f=F T$ and $t=T_{s}$ ( $T_{s}:$ scattered (measured) thermal-wave field). The main problem with this method, from the computational point of view, is that the matrix $G_{0} T_{i}$ is in many cases almost singular. To overcome this problem we used the Tykhonov regularization method [7].

\section{EXPERIMENTAL IMPLEMENTATION AND RESULTS}

An aluminum sample the dimensions of which were $3.75 \mathrm{~mm}$ wide and $2 \mathrm{~mm}$ thick was illuminated on one side with a focused laser beam at various points and a photopyroelectric pin detector was scanned along the other side. Details of the experimental set-up are given elsewhere [2]. The sample has a machined hole in its middle with diameter of $1 \mathrm{~mm}$. Fig. 2 describes the wave slice reconstruction by the current method, using only one laser position and 26 detector positions. We note the good location of the hole in both directions along with the realistic size of the defect. We also observe some edge effects (artifacts). Similar reconstructions using the ray optic approach resulted earlier [2] in imaging the defect, 
but its location was inaccurate and its presence was much less noticeable. Using a finer computational grid has resulted in a well resolved shape contour of the edge of the hole on the side of the laser locations, Fig. $2 b$.

The new method yields superior reconstructions to those obtained by ray optic approximations, and is capable of solving the Helmholtz pseudo-wave equation and reconstructing a slice tomogram of the diffusivity field.
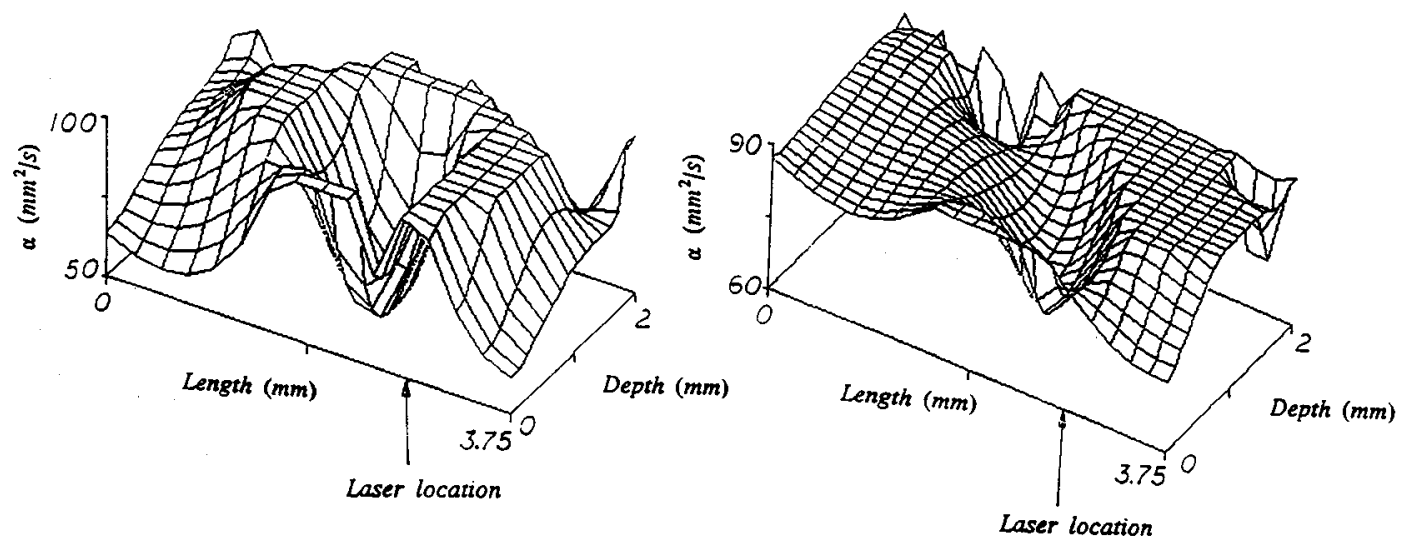

Fig. 2 a) Wave optic tomographic reconstruction of thermal diffusivity; hole at the center of the sample; b) fine computational grid, laser at $2.4 \mathrm{~mm}$ from left edge.

\section{REFERENCES}

[1] Mandelis, A. and Mieszkowski, M., A Thermal-wave sub-surface defect imaging and tomography apparatus, U.S. Patent No. 4,950,897 (August 1990).

[2] Munidasa, M. and Mandelis, A. ,J.O.S.A. A8 (1991) 1851.

[3] Munidasa, M., Mandelis, A. and Ferguson, C. ,Appl. Phys. A 54 (1992) 244.

[4] Padé, O. and Mandelis, A., Inverse Problems (In press).

[5] Mandelis, A., J. Phys. A: Math. Gen. 24 (1991) 2485.

[6] Mandelis, A., J.O.S.A. A6 (1989) 298.

[7] Hofmann, B., Regularization for Applied Inverse and Ill-Posted Problems, (Teubner, Liepzig, 1986). 